\title{
Comparison of a novel, endoscopic chest tube insertion technique versus the standard, open technique performed by novice users in a human cadaver model: a randomized, crossover, assessor-blinded study
}

Byron C. Drumheller ${ }^{1 *}$ (D), Anthony Basel ${ }^{2}$, Sakib Adnan ${ }^{3}$, Joseph Rabin ${ }^{1}$, Jason D. Pasley ${ }^{1,5}$, Jason Brocker ${ }^{1,5}$ and Samuel M. Galvagno Jr,

\begin{abstract}
Background: The technique of tube thoracostomy has been standardized for years without significant updates. Alternative procedural methods may be beneficial in certain prehospital and inpatient environments with limited resources. We sought to compare the efficacy of chest tube insertion using a novel, endoscopic device (The Reactor ${ }^{\mathrm{TM}}$ ) to standard, open tube thoracostomy.

Methods: Novice users were randomly assigned to pre-specified sequences of six chest tube insertions performed on a human cadaver model in a crossover design, alternating between the Reactor ${ }^{\mathrm{TM}}$ and standard technique. All subjects received standardized training in both procedures prior to randomization. Insertion site, which was randomly assigned within each cadaver's hemithorax, was marked by the investigators; study techniques began with skin incision and ended with tube insertion. Adequacy of tube placement (intrapleural, unkinked, not in fissure) and incision length were recorded by investigators blinded to procedural technique. Insertion time and user-rated difficulty were documented in an unblinded fashion. After completing the study, participants rated various aspects of use of the Reactor ${ }^{\mathrm{TM}}$ compared to the standard technique in a survey evaluation.
\end{abstract}

Results: Sixteen subjects were enrolled (7 medical students, 9 paramedics) and performed 92 chest tube insertions ( $n=46$ Reactor $^{\mathrm{TM}}, n=46$ standard). The Reactor ${ }^{\mathrm{TM}}$ was associated with less frequent appropriate tube positioning (41.3\% vs. $73.9 \%, P=0.0029)$, a faster median insertion time ( $47.3 \mathrm{~s}$, interquartile range $38-63.1 \mathrm{vs}$. $76.9 \mathrm{~s}$, interquartile range $55.3-106.9, P<0.0001)$ and shorter median incision length $(28 \mathrm{~mm}$, interquartile range $23-30 \mathrm{vs} .32 \mathrm{~mm}$, interquartile range $26-40, P=0.0034)$ compared to the standard technique. Using a 10-point Likert scale (1-easiest, 10hardest) participants rated the ease of use of the Reactor ${ }^{\mathrm{TM}}$ no different from the standard method (3.8 $\pm 1.9 \mathrm{vs} .4 .7 \pm 1.9$, $P=0.024)$. The Reactor ${ }^{\mathrm{TM}}$ received generally favorable scores for all parameters on the post-participation survey.

(Continued on next page)

\footnotetext{
* Correspondence: byron.drumheller@gmail.com

${ }^{1}$ Program in Trauma, Department of Surgery, R Adams Cowley Shock Trauma Center, University of Maryland School of Medicine, 22 South Greene Street, Baltimore, MD 21201, USA

Full list of author information is available at the end of the article
}

(c) The Author(s). 2018 Open Access This article is distributed under the terms of the Creative Commons Attribution 4.0 International License (http://creativecommons.org/licenses/by/4.0/), which permits unrestricted use, distribution, and reproduction in any medium, provided you give appropriate credit to the original author(s) and the source, provide a link to the Creative Commons license, and indicate if changes were made. The Creative Commons Public Domain Dedication waiver (http://creativecommons.org/publicdomain/zero/1.0/) applies to the data made available in this article, unless otherwise stated. 
(Continued from previous page)

Conclusions: In this randomized, assessor-blinded, crossover human cadaver study, chest tube insertion using the Reactor $^{\mathrm{TM}}$ device resulted in faster insertion time and shorter incision length, but less frequent appropriate tube placement compared with the standard technique. Additional studies are needed to evaluate the efficacy, safety and potential advantages of this novel device.

Keywords: Chest tubes, Trauma, Tube thoracostomy, Human cadaver, Emergency care

\section{Introduction}

The technique of tube thoracostomy has changed little since it became standard of care for the treatment of traumatic hemopneumothorax in the 1960s [1, 2]. Recent advances in minimally-invasive surgical technology and increasing awareness of complications associated with the traditional technique have led to the development of novel adaptations to the standard steps of chest tube insertion [3-7]. These new methods generally involve changes to the manner of chest wall dissection, using a laparoscopic-type trocar, or tube insertion, using a video-guided endoscopic stylet. While these sophisticated technologies may be useful in unique scenarios, they have limited applicability to many chest tube procedures, specifically those in challenging environments performed by users without extensive experience [8-10]. A practical, safe, compact and easy-to-learn novel tube thoracostomy technique can have widespread relevance across many arenas, from hospital insertion to austere pre-hospital conditions, such as the deployed environment. Furthermore, such a device would have utility for non-surgeons working in intensive care units where tube thoracostomy may be less frequently performed.

The Reactor $^{\mathrm{Tm}}$ (Sharp Medical Products, LLC., Geneva, Il) is an FDA-approved, newly designed, hand-held device created for the purposes of minimally-invasive chest tube insertion (Fig. 1) [11]. The device consists of an elliptically shaped probe with an eccentric retractable blade at the distal end and a sheath mounted over the

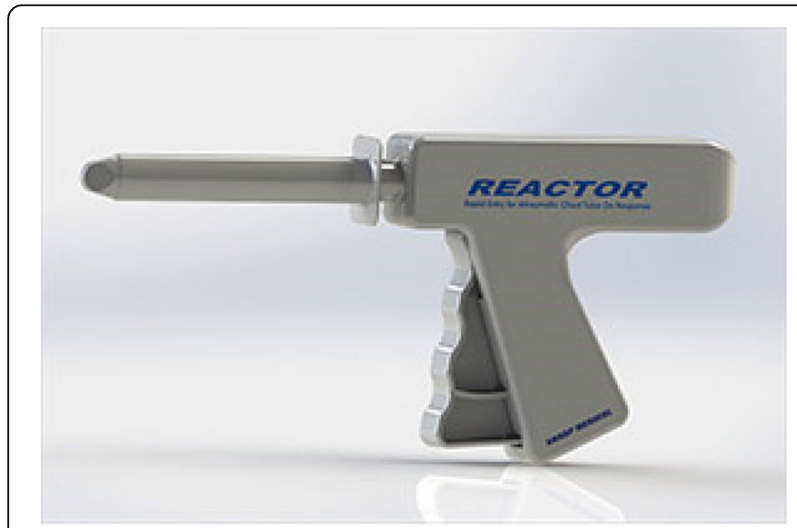

Fig. 1 The Reactor ${ }^{\mathrm{TM}}$ Endoscopic Chest Tube Device (reprinted with permission from Sharp Medical Products, LLC) proximal portion. Activated by a trigger mechanism, the blade advances and rotates with exposure of a $1 \mathrm{~mm}$ cutting edge, allowing for sharp dissection, and then rapidly and completely retracts upon release of the trigger. Pressure from the user allows for blunt dissection with the probe if the trigger is not depressed. After entrance into the pleural space, a sheath is advanced over the probe, the probe is removed, and a standard chest tube (up to 36-French) is placed through the sheath. While decompression of simulated tension pneumothorax was achieved significantly more often using the Reactor ${ }^{\mathrm{TM}}$ as compared to standard 14-gauge needle decompression in a post-mortem swine model, performance of this device in a human model is lacking [12].

We sought to evaluate the efficacy of chest tube insertion using the Reactor ${ }^{\mathrm{TM}}$ device in comparison to the standard, open technique in a randomized, controlled study using human cadavers. We chose to evaluate novice users to provide external validity to providers of this type, who perform a significant proportion of chest tube procedures in the trauma setting. We hypothesized that tube thoracostomy using the Reactor ${ }^{\mathrm{TM}}$ would be performed with a shorter incision length, faster insertion time, superior tube placement, and greater subjective ease of use compared to the conventional open technique.

\section{Methods \\ Study design and setting}

We conducted a randomized, six-period, two-treatment, crossover study comparing the Reactor ${ }^{\mathrm{TM}}$ technique to the standard tube thoracostomy technique performed by novice users on human cadavers in two sessions in December 2017 and January 2018. Cadavers were supplied by the Maryland State Anatomy Board. Sharp Medical Products, LLC. supplied the Reactor ${ }^{\mathrm{Tm}}$ devices and paid for the use of the cadavers, but was otherwise uninvolved with the study conduct, data collection, statistical analysis or publication of the results.

\section{Participants}

We recruited first and second year medical students from the University of Maryland School of Medicine and national registered paramedics certified in the state of Maryland. Voluntary participation was sought through e-mail advertisements, flyers, and announcements made 
in medical school and emergency medical services interest groups. Subjects were required to have never performed chest tube placement on a live patient but could have performed prior procedures using manikin, animal or human cadaver models.

\section{Randomization and treatment sequence assignment}

Each study participant was assigned to perform a total of six chest tube insertions, three using the Reactor ${ }^{\text {Tx }}$ technique and three using the standard technique, completed in a pre-specified alternating order. Since the subjects were all novice users, we anticipated that procedural competency could increase during the study from the first to the sixth chest tube insertion. To achieve an equal total number of insertions using each of the two techniques and an equal probability of each technique being performed for the first through sixth procedure, a study-order matrix was designed (Table 1). Using a computer-generated random number sequence, subjects were randomly assigned to a particular sequence of chest tube insertions. Unblinded assignment was performed by one group of study investigators who later performed the unblinded portion of data collection. Treatment allocation was blinded, however, to those investigators that later ascertained the primary outcome.

\section{Cadaver model}

Tube thoracostomy was performed on fresh, nonembalmed human cadavers, stored at $4{ }^{\circ} \mathrm{C}$ and allowed to warm to room temperature prior to use. Prior to study initiation, a median sternotomy was performed and both medial pleural borders were widely opened to allow for visual and tactile assessment of each hemithorax. Two to three pairs of twoinch eye screws were inserted into each half of the sternum and secured together with rubber bands to reapproximate the shape of the thoracic cavity (Fig. 2a). The cadavers were positioned supine with corpse elevators beneath both scapula and with the arms abducted to 90 degrees.

To simulate realistic conditions in which novice users may not correctly identify the ideal insertion site for chest tube placement (anterior-mid axillary line, 4th or 5th intercostal space) $[13,14]$, we divided each hemithorax into ten potential insertion sites (corresponding roughly to regions between the anterior and posterior axillary lines and from the 3rd to 6th intercostal spaces). Each location was labeled in colored marker visible only under ultraviolet-light (Fig. 2b). Prior to randomization of the study participants, we assigned each pre-specified chest tube procedure in the study-order matrix (each cell in Table 1) to a unique insertion site on one hemithorax of one of the cadavers. For each hemithorax of each cadaver, a separate order of insertion sites was created using a computerized random number sequence from 1 to 10 . For each study subject, starting with their first procedure the location of each tube insertion was assigned to a site on a cadaver based on the previously generated sequence for that cadavers' hemithorax. For simplicity, the first procedure for each participant was always performed on the right hemithorax, with subsequent procedures alternating sides. Once all ten sites on a given hemithorax for a given cadaver were assigned, the following assignment proceeded to the next available cadaver, maintaining the order of laterality, using the unique random sequence of insertion sites linked to that cadaver's hemithorax. Each insertion site was unique to one chest tube procedure; no locations were used more than once.

Unblinded study investigators utilized an ultraviolet flashlight to identify and drape the appropriate insertion location with surgical towels prior to each chest tube insertion. The combination of draping and ultraviolet-only visible marking was intended to blind the participants to the exact location on the cadaver at which they were performing each tube insertion.

\section{Interventions}

All subjects received a protocolized 2-h training session prior to randomization. Instructional videos and concomitant narration of the standard and Reactor ${ }^{\mathrm{Tm}}$ techniques were shown and the participants completed one chest tube insertion using each technique with real-time teaching by the study investigators. These procedures

Table 1 Study-Order sequence assignment matrix

\begin{tabular}{|c|c|c|c|c|c|c|c|c|c|c|c|c|c|c|c|c|}
\hline \multirow[b]{2}{*}{ Method } & \multicolumn{16}{|c|}{ Subject } \\
\hline & (1) & (2) & (3) & (4) & (5) & (6) & (7) & (8) & (9) & (10) & (11) & (12) & (13) & (14) & (15) & (16) \\
\hline $\mathrm{R}$ & 4 & 5 & 6 & 1 & 2 & 3 & 4 & 5 & 6 & 1 & 2 & 3 & 4 & 5 & 6 & 1 \\
\hline S & 5 & 6 & 1 & 2 & 3 & 4 & 5 & 6 & 1 & 2 & 3 & 4 & 5 & 6 & 1 & 2 \\
\hline $\mathrm{R}$ & 6 & 1 & 2 & 3 & 4 & 5 & 6 & 1 & 2 & 3 & 4 & 5 & 6 & 1 & 2 & 3 \\
\hline$S$ & 1 & 2 & 3 & 4 & 5 & 6 & 1 & 2 & 3 & 4 & 5 & 6 & 1 & 2 & 3 & 4 \\
\hline $\mathrm{R}$ & 2 & 3 & 4 & 5 & 6 & 1 & 2 & 3 & 4 & 5 & 6 & 1 & 2 & 3 & 4 & 5 \\
\hline S & 3 & 4 & 5 & 6 & 1 & 2 & 3 & 4 & 5 & 6 & 1 & 2 & 3 & 4 & 5 & 6 \\
\hline
\end{tabular}

$R$ Reactor technique, $S$ Standard technique; (Numbers) represent individual study participants; Numbers below each labelled study participant in each column represent the order of chest tube insertion $(1=$ first, $6=$ sixth $)$ 

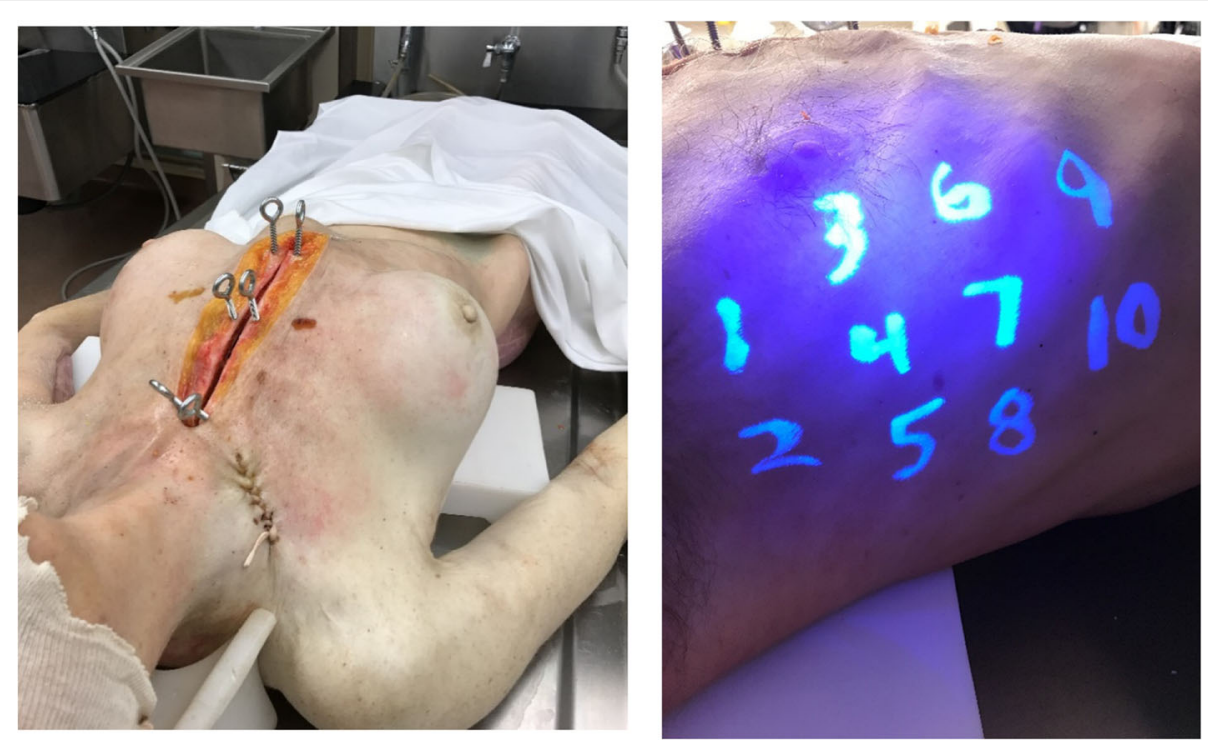

Fig. 2 Human Cadaver Model. a (left) demonstrates the setup of the median sternotomy used to evaluate tube placement. b (right) displays the numbering system used to mark the pre-specified locations to chest tube insertion

were performed on cadavers specifically used for training and not later utilized for the study implementation.

All chest tube insertions were performed using a 10 or 11-blade scalpel and a 32-French chest tube (Atrium Medical Corporation, Hudson, NH). Subjects were instructed to aim for placement in the posterior apex of the pleural cavity, corresponding to the customary desired position in the setting of undifferentiated thoracic trauma, and to avoid the neurovascular bundle by inserting the tube just over the rib.

For the standard tube thoracostomy technique, one 5.5-in. curved Kelly clamp and one 8-in. curved Kelly clamp were provided. Subjects were instructed in the steps of the standard technique as follows: 1) make a $\sim 3 \mathrm{~cm}$ anteroposterior oriented incision through the skin, 2) using the 5.5-in. curved Kelly clamp blunt dissect through the subcutaneous tissue and enter the pleural space, 3) enlarge the pleurotomy in the anteroposterior direction by spreading the jaws of the clamp, 4) insert one finger into the pleural space and palpate the positioning deep to the ribs within the pleural cavity, 5) insert the chest tube using either the 8-in. curved Kelly clamp or one finger to guide the tip of the tube into the desired location.

For the Reactor ${ }^{\mathrm{mu}}$ technique, only the endoscopic device was provided in addition to the scalpel and chest tube. Subjects were instructed in the steps of the Reactor ${ }^{\mathrm{rm}}$ technique as follows: 1) make a $\sim 2 \mathrm{~cm}$ anteroposterior oriented incision through the skin, 2) place the tip of the device into the incision and, applying constant pressure, repeatedly depress the trigger mechanism to dissect through the subcutaneous tissue and enter the pleural space, 3) slide the sheath over the device barrel into the pleural space and then remove the device barrel, 4) insert the chest tube through the sheath, directing the angle/location as desired by manipulating the sheath, 5) remove the sheath over the chest tube. If the barrel or sheath of the device were unable to smoothly pass through the skin incision, participants were instructed to elongate the skin incision using the scalpel.

Procedures were observed by unblinded study investigators to ensure compliance with the assigned technique. Both techniques were considered complete when the subject indicated they were satisfied with the tube placement. The tube was not sutured to the skin nor connected to a negative pressure drainage system. After performing each two chest tube procedures, one on either side of the cadaver, the subjects stepped out of the lab while blinded study data collection was performed by a separate team of assessors. Following the blinded assessments, subjects returned and performed two more insertions, repeating these steps for a total of six insertions per participant.

\section{Data collection and outcome assessment}

The primary outcome measure was successful tube placement, defined as a chest tube meeting all the following criteria: 1) distal tip and at least one side hole within the pleural space, 2) not terminating in the major fissure, 3) not kinked. All the side holes were not required to be within the pleural space to satisfy criteria 1 ), however the tube could not pass through the pleural space and then out of the pleural space (e.g. through the diaphragm) for criteria 1) to be satisfied. If a portion of the tube passed through the major fissure but at least one side hole at the distal aspect was outside of the 
fissure, the tube was considered to satisfy criteria 2). A kink was defined as an abrupt change in direction that resulted in $\mathrm{a} \geq 90$-degree angle deviation in tube shape.

Study investigators blinded to insertion technique measured and recorded tube position after every 2 procedures (one on each hemithorax of the cadaver), with study participants not present. The rubber bands connecting the eye screws placed in the cadaver's sternum were removed and the sternotomy was retracted, allowing access to bilateral pleural cavities. Tube position was determined by manual palpation of the course of the tube from entrance into the pleural space to its distal tip, with the aid of visualization if needed. After position was determined, the tubes were removed and the sternum was reapproximated. This procedure was repeated for every pair of tube insertions.

Secondary outcomes included: skin incision length (millimeters), measured using a ruler by the blinded investigators, procedure time from start of incision to satisfactory tube placement (seconds), measured with a stopwatch by the unblinded study investigators, and subjective level of difficulty of each tube insertion (1-10 on a Likert scale, 1 being easiest, 10 being most difficult) rated by the participants after each procedure and recorded by the unblinded study investigators. A nine-question survey was distributed upon completion of the study to evaluate participants' impressions of the Reactor ${ }^{\mathrm{Tm}}$ compared to the conventional open technique. Participant type (medical student or paramedic) and prior experience with simulated chest tube placement was also documented.

\section{Sample size calculation and statistical analysis}

The study was initially powered to satisfy the sample size requirement for a primary outcome of incision size. The hypothesis was originally that an incision size of less than $50 \%(2 \mathrm{~cm}$ vs. $4 \mathrm{~cm})$ would be observed for chest tube insertion with the Reactor ${ }^{\mathrm{m}}$. Assuming $80 \%$ power with an alpha level of 0.05 and standard deviation of 1 $\mathrm{cm}$, only 8 participants would be required. However, prior to study initiation, a more clinically relevant outcome was considered. For the outcome of proportion of correct chest tube placements, a two-sample paired-proportions test was used, assuming $80 \%$ power and an alpha level of 0.05 . With a hypothesized success rate of $80 \%$ correct placement with the standard open technique, a sample size of 15 subjects was calculated.

Descriptive statistics were performed with use of the Wilcoxon rank sum test for non-parametrically distributed continuous data and the Student's t-test for parametrically distributed continuous data. Histograms, q-q plots, and the Shapiro-Wilk test were used to assess normality of continuous data. The Fisher's exact test was used to analyze categorical data given the relatively small sample size. All tests were two-tailed, and a relatively stringent $P$ value of $<0.005$ was considered statistically significant $[15,16]$. All analyses were performed using Stata version 15.1 (StataCorp, College Station, TX, USA) and GraphPad Prism version 7.0d for Mac (GraphPad Software, La Jolla, CA, USA).

\section{Results}

Sixteen subjects participated in the study, 7 (44\%) were first- or second-year medical students and $9(56 \%)$ were nationally registered paramedics. Twelve (75\%) subjects had no prior experience with simulated chest tube placement and 4 (25\%) had performed one or more simulated procedures. Fourteen $(87.5 \%)$ subjects performed 6 chest tube insertions ( 3 Reactor $^{\mathrm{m}}$, 3 standard), while 2 subjects (12.5\%) only completed 4 chest tube procedures (2 Reactor $^{\mathrm{rm}}, 2$ standard) due to limited anatomy lab availability. In total, 92 chest tube insertions (46 Reactor ${ }^{\mathrm{Tm}}$, 46 standard) were performed by participants, all using the pre-assigned order/technique.

Unadjusted outcomes are presented in Table 2. The primary outcome, frequency of successful tube placement, occurred significantly more often using the standard technique compared with the Reactor ${ }^{\text {Tx }}(73.9 \%$ vs. $41.3 \%, p=0.0029)$. The individual components of successful tube placement favored the standard technique but were not significantly different by themselves between groups. In total, 9 chest tubes $(2$ standard, 7 Reactor $\left.^{\mathrm{mm}}\right)$ were not placed within the pleural space. The 2 extra-pleural tubes in the standard group were entirely within the subcutaneous space, 5 of the extra-pleural Reactor $^{\text {Tax }}$ tubes were fully subcutaneous and 2 of the extra-pleural Reactor ${ }^{\text {ra }}$ tubes entered the pleural space but then coursed through the diaphragm into the liver. The Reactor $^{\text {Tx }}$ was associated with statistically significantly faster median insertion time $(\sim 30 \mathrm{~s})$ and shorter incision length $(\sim 4 \mathrm{~mm})$ compared with the standard technique. Study participants' rating of the overall difficulty of tube placement was not different between groups.

Over successive attempts, insertion time remained significantly faster in the Reactor ${ }^{\mathrm{rm}}$ group compared to the open technique (Fig. 3), while difference in incision length was inconsistently significantly shorter with the Reactor ${ }^{\mathrm{m}}$ device (Fig. 4). The frequency of successful tube placement was also inconsistently greater in the standard technique group over repeated attempts (Fig. 5).

Results from the end-of-study survey are shown in Fig. 6; the Reactor ${ }^{\text {tm }}$ received generally favorable scores for all parameters.

\section{Discussion}

In this randomized, assessor-blinded, crossover cadaver study among novice participants, use of the Reactor ${ }^{\text {rat }}$ device for chest tube insertion resulted in significantly less 
Table 2 Unadjusted outcomes

\begin{tabular}{llll}
\hline Outcome & Standard Technique $N=46$ & Reactor $^{\mathrm{Tm}}$ Technique $N=46$ & $P$-value \\
\hline Successful tube placement, n (\%) & $34(73.9)$ & $19(41.3)$ & $39(84.8)$ \\
$\quad$ Correctly placed in pleural space, $\mathrm{n}(\%)$ & $44(95.6)$ & $14(30.4)$ & 0.0029 \\
$\quad$ Kinked tube, $\mathrm{n}(\%)$ & $8(17.4)$ & $7(15.2)$ & 0.158 \\
$\quad$ Placed in fissure, $\mathrm{n}(\%)$ & $3(6.5)$ & $47.3(38-63.1)$ & 0.051 \\
Insertion time (sec) Median (IQR) & $76.9(55.3-106.9)$ & $28(23-30)$ & 0.075 \\
Incision length (mm) Median (IQR) & $32(26-40)$ & $3.8(1.9)$ & 0.0034 \\
Perceived difficulty (1-10 scale; 10 most difficult) Mean (SD) & $4.7(1.9)$ & & 0.024 \\
\hline
\end{tabular}

IQR interquartile range, $S D$ standard deviation

frequent successful tube placement, but was associated with a faster insertion time and shorter incision length compared with the standard, open technique. Participants rated the ease of use of Reactor ${ }^{\mathrm{Tm}}$ more favorably compared to the standard open technique, but using a stringent statistical cut-off (i.e., a $P$ value $<0.005$ ), these results were not statistically significant.

For providers in prehospital, military, or intensive care settings, pneumothorax and hemothorax are potentially lethal conditions and must be treated promptly [2, 9, 17]. Unfortunately, both needle decompression and tube thoracostomy are infrequently applied by many non-surgeons in these environments, and a high rate of failure has been observed with the classic technique of needle decompression for tension pneumothorax [18-20]. A compact, efficacious, easy-to-use device that requires less technical skill may be appealing for these scenarios. One prior animal study has described the use of a laparoscopic-type trocar for decompression of tension pneumothorax and found the technique to be more effective than standard needle decompression [21]. Furthermore, Kuckelman et al. showed that the Reactor ${ }^{\text {rix }}$ was significantly more successful at achieving rapid and complete resolution of tension pneumothorax compared to needle decompression ( $88 \%$ vs. $44 \%, P<0.001)$ in a post-mortem swine model.

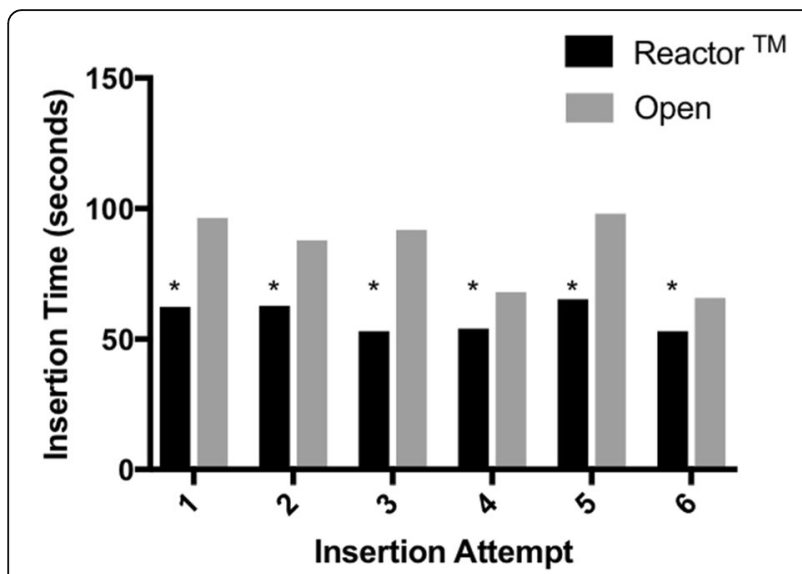

Fig. 3 Insertion Time for the Reactor ${ }^{\mathrm{TM}}$ vs. Standard Technique Over the Course of Successive Attempts. ${ }^{*} P<0.005$
Laparoscopic-type trocar procedures have been reported in two human case series for the treatment of simple pneumothoraces in which a chest tube is subsequently inserted through the trocar $[4,5]$. Nevertheless, tube thoracostomy using a trocar-type device or the Reactor ${ }^{\mathrm{ma}}$ has not yet been compared with the standard, open technique.

The Reactor ${ }^{\mathrm{Tn}}$ is a novel device with several purported design advantages for the performance of tube thoracostomy. The device uses a rotating and retracting blade that "carves" rather than punctures the subcutaneous layer, potentially resulting in less tissue damage. The oval shaft is designed to fit between ribs, potentially avoiding intercostal vascular or nerve injuries. Medium to large chest tubes can be inserted with potentially shorter incisions due to a "tunneling" effect created by the device during subcutaneous dissection. Importantly, the device has the potential to decrease the incision length required for tube insertion and can be performed rapidly, thereby potentially resulting in less patient discomfort. These design features may be especially advantageous for less experienced providers tasked with performing chest tube insertion in a variety of settings.

In this study, novice proceduralists confirmed some of the design advantages (shorter incision length, faster insertion time) of the Reactor ${ }^{\text {Tx }}$ in a cadaver model. Given the high prevalence of pain, pulmonary complications, and morbidity associated with both blunt and penetrating thoracic trauma, novel techniques that can lessen the discomfort associated with tube thoracostomy are highly desirable. The need for local and parenteral analgesia during tube thoracostomy placement-including opioids-is substantial [22]. In order to truly assess pain and analgesic needs with the Reactor ${ }^{\mathrm{rm}}$ compared to the standard technique and to evaluate the clinical significance of the small differences in insertion time and incision length we found, a human study would be needed using validated measures for assessment of pain.

By contrast, the primary outcome in this study-appropriate chest tube placement-was less frequently achieved in the Reactor $^{\text {Ta }}$ group compared to the standard technique. The potentially life-threatening nature of the conditions treated by tube thoracostomy and the significant 

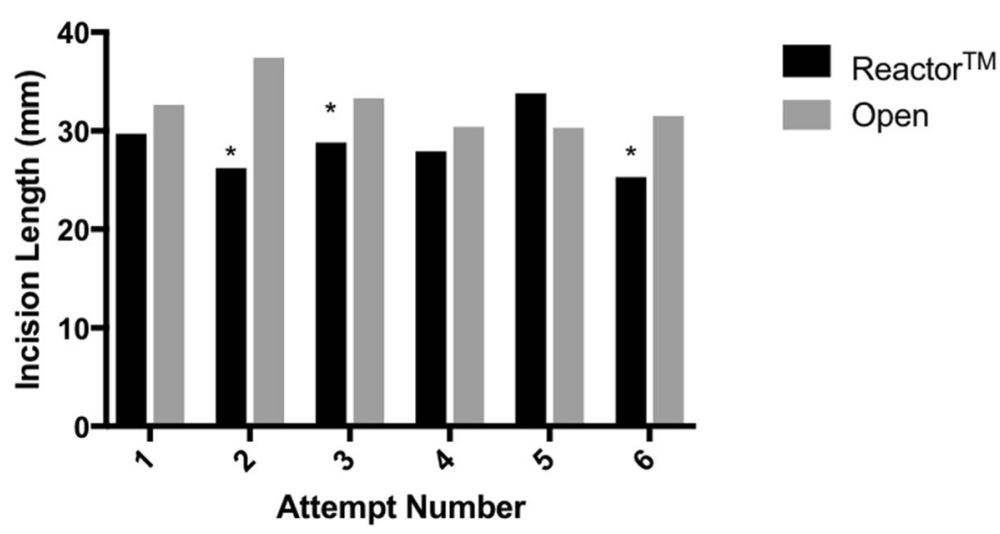

Attempt Number

Fig. 4 Mean Incision Length for the Reactor ${ }^{\mathrm{TM}}$ vs. Standard Technique Over the Course of Successive Attempts. ${ }^{*} P<0.005$

harm that can result from malpositioned chest tubes necessitates that any new procedural technique has at least equivalent safety and efficacy compared to the standard, open procedure [3, 10]. Our results suggest this may not be the case with the Reactor ${ }^{\mathrm{Tm}}$. Evaluation of the occurrence of the individual criteria for successful tube placement suggests the difference between groups was primarily due to more frequent kinking and

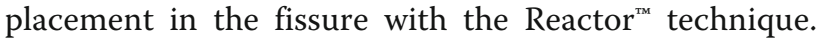
This could be because chest tubes placed using the Reactor $^{\mathrm{Tm}}$ are inserted into the thoracic cavity through the short introducer sheath, which limits tactile feedback on the initial path of the tube compared to the open technique, where a finger can be inserted into the chest to guide the tube in a particular direction. Extra-pleural tube placement also occurred in 7/46 (15\%) Reactor $^{\text {TM }}$ procedures compared to $2 / 46$ (4\%) insertions using the standard technique. We purposely manipulated site selection to include locations predisposed to more frequent complications to simulate potential application by novice users, since prior data has shown an $\sim 5 \%$ rate of extrapleural tube placement and as high as a $60 \%$ rate of placement outside of the ideal location (4th or 5th intercostal space) when chest tubes are placed by trainees under emergent scenarios $[13,14]$. A notable limitation of the Reactor ${ }^{\mathrm{TM}}$ technique is that it does not allow for confirmation of entrance into the pleural cavity through insertion of a finger into the chest and palpation of the inner surface of the ribs. Though our study was not powered to show a significant difference in the frequency of extra-pleural tube placement, the overall results suggest that further research is needed to critically evaluate the safety of the Reactor ${ }^{\mathrm{Tm}}$ device.

Our study has several strengths, including the randomized, crossover design, blinded primary outcome assessment, and recruitment of a population of novice users from different backgrounds. Conversely, our results may not be generalizable to more technically experienced providers and are inherently limited by the nature of the human

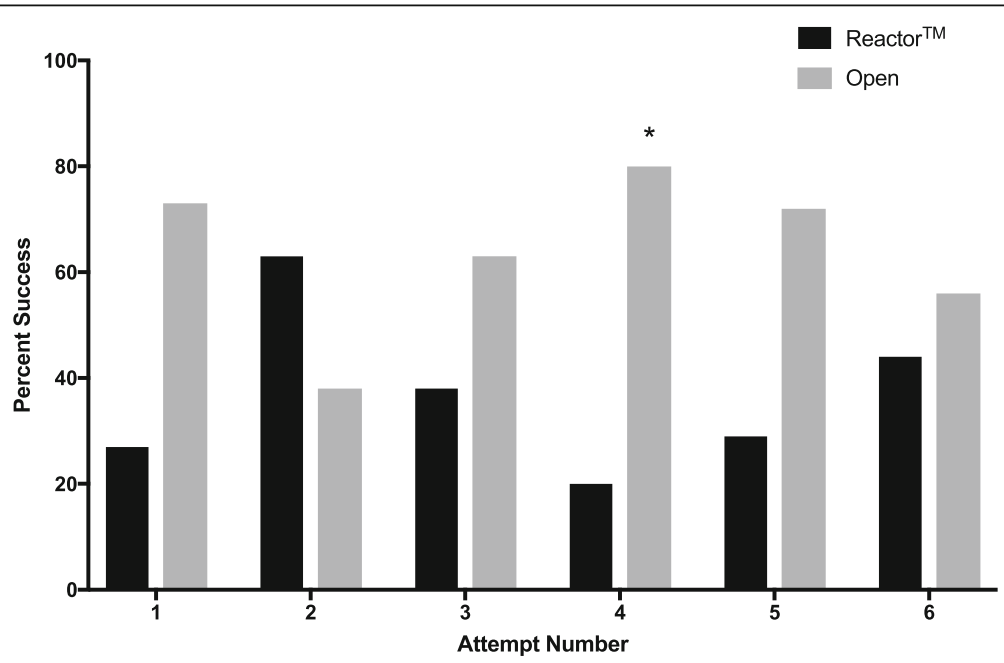

Fig. 5 Percentage of Successful Tube Placements for the Reactor ${ }^{\mathrm{TM}}$ vs. Standard Technique Over the Course of Successive Attempts. ${ }^{*} P<0.005$ 


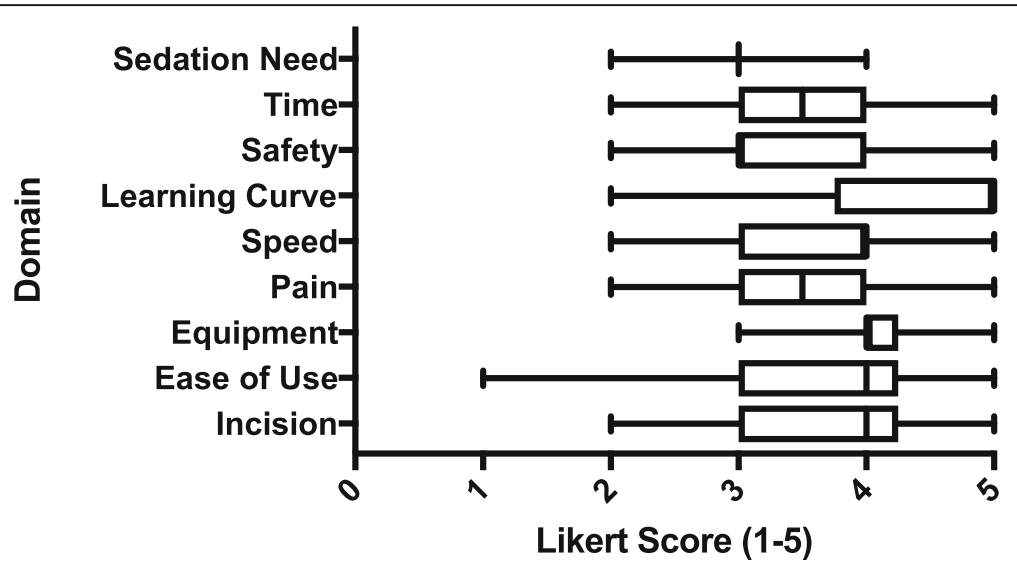

Fig. 6 Results of Post-participation Survey. A Likert scale of 1 (strongly disagree) to 5 (strongly agree) was used to assess various subjective parameters comparing the Reactor ${ }^{\mathrm{TM}}$ to the standard technique. Survey key: Compared to the open insertion technique, the Reactor ${ }^{\mathrm{TM}}$ device: 1. (Incision) Is associated with a smaller incision? 2. (Ease of Use) Is easier to use? 3. (Equipment) Requires less equipment? 4. (Pain) Is likely to cause LESS pain for the patient? 5. (Speed) Allows for a chest tube to be inserted more rapidly? 6. (Learning Curve) Is NOT as difficult to learn? 7. (Safety) Improves clinician safety (i.e., needle sticks, injuries, etc.)? 8. (Time) Requires LESS time to insert a chest tube? 9. (Sedation Need) Is likely to require LESS sedation and analgesia?

cadaver model. We observed a higher than expected frequency of tube misplacement among both techniques, emphasizing the significant learning curve for chest tube placement. Tube thoracostomy is an advanced skill that requires practice, experience, and proficiency, regardless of the type of insertion technique.

\section{Conclusions}

In summary, this randomized, assessor-blinded, crossover cadaver study comparing the Reactor ${ }^{\text {rm }}$ to standard tube thoracostomy technique in novice participants showed chest tube insertion was more frequently successful with the standard, open technique but slightly faster and associated with a shorter incision length using the Reactor $^{\text {Tix }}$ device. Further research is needed to scrutinize the safety and efficacy of the Reactor ${ }^{\text {mi }}$ device and investigate whether it has clinically significant benefits over the standard technique with regard to insertion time, incision length, and procedural discomfort when used in patients.

\section{Abbreviation}

FDA: Food and Drug Administration

\section{Acknowledgements}

We would like to thank Anthony Pleasant, Ronald Wade and the staff at the Maryland State Anatomy Board for their help with performance of the study. We also thank David Gens, MD, FACS for the use of surgical instruments and chest tube equipment. This study would not have been possible without the participation of the study participants, and we thank them for their contribution of time and expertise. Finally, we could not have conducted the study without the generous and munificent donation of the human cadavers and are forever indebted to the individuals and their families that made this possible.

\section{Funding}

This work was partially sponsored by Sharp Medical Products, LLC, who provided funding for the Reactor ${ }^{\mathrm{T}} \mathrm{M}$ devices and human cadaver use. No sponsors were involved in the collection, management or analysis of the data; preparation of the manuscript; or the decision to submit the manuscript for publication.

\section{Availability of data and materials} Not applicable.

\section{Authors' contributions}

$B C D, J P$, JB, and SMG designed the study. SMG obtained funding for the project. BCD, SA, and JR. performed blinded data collection; AB. and SMG. performed unblinded data collection. BCD and SMG performed the data analysis. $B C D$ and $A B$. drafted the manuscript and all authors contributed to critical revision. $B C D$ and $S M G$ are responsible for the overall conduct of the study. All authors read and approved the final manuscript.

\section{Authors' information}

The content is the sole responsibility of the authors and is not to be construed as official or as reflecting the views of any sponsors. The views expressed in this paper are those of the authors and do not reflect the official policy or position of the Department of the Air Force, Department of Defense, or the U.S. Government.

\section{Ethics approval and consent for participate}

The study was approved by the Institutional Review Board at the University of Maryland School of Medicine. Informed consent was obtained from all participants.

Consent for publication

Not applicable.

\section{Competing interests}

The authors declare that they have no competing interests.

\section{Publisher's Note}

Springer Nature remains neutral with regard to jurisdictional claims in published maps and institutional affiliations. 


\section{Author details}

${ }^{1}$ Program in Trauma, Department of Surgery, R Adams Cowley Shock Trauma Center, University of Maryland School of Medicine, 22 South Greene Street, Baltimore, MD 21201, USA. ${ }^{2}$ Division of Critical Care Medicine, Department of Medicine, University of Maryland School of Medicine, 22 South Greene Street, Baltimore, MD 21201, USA. ${ }^{3}$ School of Medicine, University of Maryland School of Medicine, 22 South Greene Street, Baltimore, MD 21201, USA. ${ }^{4}$ Division of Critical Care Medicine, Department of Anesthesiology, University of Maryland School of Medicine, 22 South Greene Street, Baltimore, MD 21201, USA. ${ }^{5}$ United States Air Force Center for Trauma and Readiness Sustainment (CSTARS)-Baltimore, University of Maryland School of Medicine, 22 South Greene Street, Baltimore, MD 21201, USA.

Received: 31 August 2018 Accepted: 29 November 2018

Published online: 27 December 2018

\section{References}

1. Monaghan SF, Swan KG. Tube thoracostomy: the struggle to the "standard of care". Ann Thorac Surg. 2008;86(6):2019-22.

2. $\quad$ Fitzgerald M, Mackenzie CF, Marasco S, Hoyle R, Kossmann T. Pleura decompression and drainage during trauma reception and resuscitation. Injury. 2008;39(1):9-20.

3. Hernandez MC, El Khatib M, Prokop L, Zielinski MD, Aho JM. Complications in Tube Thoracostomy: Systematic review and meta-analysis. J Trauma Acute Care Surg. 2018;85(2):410-6.

4. Dural K, Gulbahar G, Kocer B, Sakinci U. A novel and safe technique in closed tube thoracostomy. J Cardiothorac Surg. 2010;5:21.

5. Nasr MM. Closed-tube thoracostomy: a novel emergency surgery technique. Surg Laparosc Endosc Percutan Tech. 2014;24(4):342-4.

6. Chen $\mathrm{CH}$, Chang H, Lee SY, Liu HC, Hung TT, Tai CY. Video-guided tube thoracostomy with use of an electrical nonfiberoptic endoscope. Ann Thorac Surg. 2013;96(4):1450-4

7. Hernandez MC, Vogelsang D, Anderson JR, Thiels CA, Beilman G, Zielinski $M D$, et al. Visually guided tube thoracostomy insertion comparison to standard of care in a large animal model. Injury. 2017:48(4):849-53.

8. Ball CG, Lord J, Laupland KB, Gmora S, Mulloy RH, Ng AK, et al. Chest tube complications: how well are we training our residents? Can J Surg. 2007;50(6):450-8.

9. Gerhardt RT, Berry JA, Blackbourne LH. Analysis of life-saving interventions performed by out-of-hospital combat medical personnel. J Trauma. 2011;71(1 Suppl):S109-13.

10. Davis JS, Garcia GD, Jouria JM, Wyckoff MM, Alsafran S, Graygo JM, et al. Identifying pitfalls in chest tube insertion: improving teaching and performance. J Surg Educ. 2013;70(3):334-9.

11. Sharp Medical Reactor Chest Tube Device Instructions for Use. Geneva, Illinois: Sharp Medical Products, LLC.; 2015.

12. Kuckelman J, Derickson M, Phillips C, Barron M, Marko S, Eckert M, et al. Evaluation of a novel thoracic entry device versus needle decompression in a tension pneumothorax swine model. Am J Surg. 2018;215(5):832-5.

13. Hernandez MC, Laan DV, Zimmerman SL, Naik ND, Schiller HJ, Aho JM. Tube thoracostomy: increased angle of insertion is associated with complications. J Trauma Acute Care Surg. 2016:81(2):366-70.

14. Benns MV, Egger ME, Harbrecht BG, Franklin GA, Smith JW, Miller KR, et al. Does chest tube location matter? An analysis of chest tube position and the need for secondary interventions. J Trauma Acute Care Surg. 2015;78(2): 386-90.

15. Wasserstein RL, Lazar NA. The ASA's statement on p-values: context, process, and purpose. Am Stat. 2016;70(2):129-33.

16. loannidis JPA. The proposal to lower P value thresholds to .005. JAMA. 2018; 319(14):1429-30

17. Lairet JR, Bebarta VS, Burns CJ, Lairet KF, Rasmussen TE, Renz EM, et al. Prehospital interventions performed in a combat zone: a prospective multicenter study of 1,003 combat wounded. J Trauma Acute Care Surg. 2012;73(2 Suppl 1):S38-42.

18. Ball CG, Wyrzykowski AD, Kirkpatrick AW, Dente CJ, Nicholas JM, Salomone $J P$, et al. Thoracic needle decompression for tension pneumothorax: clinical correlation with catheter length. Can J Surg. 2010;53(3):184-8.

19. Aho JM, Thiels CA, El Khatib MM, Ubl DS, Laan DV, Berns KS, et al. Needle thoracostomy: clinical effectiveness is improved using a longer angiocatheter. J Trauma Acute Care Surg. 2016;80(2):272-7.
20. Davis DP, Pettit K, Rom CD, Poste JC, Sise MJ, Hoyt DB, et al. The safety and efficacy of prehospital needle and tube thoracostomy by aeromedical personnel. Prehosp Emerg Care. 2005;9(2):191-7.

21. Hatch Q, Debarros M, Johnson E, Inaba K, Martin M. Standard laparoscopic trocars for the treatment of tension pneumothorax: a superior alternative to needle decompression. J Trauma Acute Care Surg. 2014;77(1):170-5.

22. Galvagno SM Jr, Smith CE, Varon AJ, Hasenboehler EA, Sultan S, Shaefer G, et al. Pain management for blunt thoracic trauma: a joint practice management guideline from the eastern Association for the Surgery of trauma and trauma anesthesiology society. J Trauma Acute Care Surg. 2016;81(5):936-51.
Ready to submit your research? Choose BMC and benefit from:

- fast, convenient online submission

- thorough peer review by experienced researchers in your field

- rapid publication on acceptance

- support for research data, including large and complex data types

- gold Open Access which fosters wider collaboration and increased citations

- maximum visibility for your research: over $100 \mathrm{M}$ website views per year

At BMC, research is always in progress.

Learn more biomedcentral.com/submissions 